\title{
Comparison of Intrafractional Motion in Head and Neck Cancer Between Two Immobilization Methods During Stereotactic Ablative Radiation Therapy by CyberKnife
}

This article was published in the following Dove Press journal:

Cancer Management and Research

\author{
Chen-Lin Kang ${ }^{1-3}$ \\ Tsair-Fwu Lee iD ${ }^{1,4,5}$ \\ Shan-Ho Chan ${ }^{2}$ \\ Shyh-Chang Liu ${ }^{3}$ \\ Jui-Chu Wang ${ }^{6}$ \\ Cheng-Hsiang Tsai ${ }^{1}$ \\ Kuan-Cho Liao' \\ Fu-Min Fang ' \\ Liyun Chang $^{7}$ \\ Chun-Chieh Huang (iD) ${ }^{1,8}$ \\ 'Department of Radiation Oncology, \\ Kaohsiung Chang Gung Memorial Hospital \\ and Chang Gung University College of \\ Medicine, Kaohsiung, Taiwan; ${ }^{2}$ Department \\ of Medical Imaging and Radiology, Shu-Zen \\ Junior College of Medicine and \\ Management, Kaohsiung, Taiwan; \\ ${ }^{3}$ Department of Information Engineering, \\ I-Shou University, Kaohsiung, Taiwan; \\ ${ }^{4}$ Medical Physics and Informatics \\ Laboratory of Electronics Engineering, \\ National Kaohsiung University of Science \\ and Technology, Kaohsiung, Taiwan; \\ ${ }^{5}$ Biomedical Engineering, Kaohsiung \\ Medical University, Kaohsiung, Taiwan; \\ ${ }^{6}$ Department of Anatomical Pathology, \\ Kaohsiung Chang Gung Memorial Hospital \\ and Chang Gung University College of \\ Medicine, Kaohsiung, Taiwan; ${ }^{7}$ Department \\ of Medical Imaging and Radiological \\ Sciences, I-Shou University, Kaohsiung, \\ Taiwan; ${ }^{8} \mathrm{Graduate}$ Institute of Clinical \\ Medical Sciences, College of Medicine, \\ Chang Gung University, Taoyuan, Taiwan
}

Correspondence: Chun-Chieh Huang Department of Radiation Oncology, Kaohsiung Chang Gung Memorial

Hospital, No 123, Dapi Road, Niaosong

Dist., Kaohsiung 833, Taiwan

Tel +886975056110

Fax +88677322813

Email cgukinace@gmail.com
Purpose: Maintaining immobilization to minimize spine motion is very important during salvage stereotactic ablative radiation therapy (SABR) for recurrent head and neck cancer. This study aimed to compare the intrafractional motion between two immobilization methods.

Patients and Methods: With a spine tracking system for image guiding, 9094 records from 41 patients receiving SABR by CyberKnife were obtained for retrospective comparison. Twenty-one patients were immobilized with a thermoplastic mask and headrest (Group A), and another 20 patients used a thermoplastic mask and headrest together with a vacuum bag to support the head and neck area (Group B). The intrafractional motion in the X (superiorinferior), Y (right-left), Z (anterior-posterior) axes, 3D (three-dimensional) vector, Roll, Pitch and Yaw in the two groups was compared. The margins of the planning target volume (PTV) to cover $95 \%$ intrafractional motion were evaluated.

Results: The translational movements in the X-axis, Y-axis, and 3D vector in Group A were significantly smaller than in Group B. The rotational errors in the Roll and Yaw in Group A were also significantly smaller than those in Group B; conversely, those in the Pitch in Group A were larger. To cover 95\% intrafractional motion, margins of $0.96,1.55$, and $1.51 \mathrm{~mm}$ in the $\mathrm{X}, \mathrm{Y}$ and $\mathrm{Z}$ axes, respectively were needed in Group A, and 1.06, 2.86, and $1.34 \mathrm{~mm}$, respectively were required in Group B.

Conclusion: The immobilization method of thermoplastic mask and head rest with vacuum bag did not provide better immobilization than that without vacuum bag in most axes. The clinical use of $2 \mathrm{~mm}$ as a margin of PTV to cover $95 \%$ intrafractional motion was adequate in Group A but not in Group B.

Keywords: stereotactic ablative radiation therapy, CyberKnife, intrafractional motion, immobilization method, head and neck cancer

\section{Introduction}

In recent years, salvage stereotactic ablative radiation therapy (SABR) has shown a potential for recurrent head and neck cancer, providing patients with a chance to control tumors. Due to the structural complexity of the head and neck, errors can lead to underdosing of the target, while organs at risk may receive excessive radiation doses. Head and neck cancer is particularly susceptible to underdosing due to changes in position, resulting in poor therapeutic outcomes. ${ }^{1,2}$ Although the 
patient uses a thermoplastic mask and head support, the position of the neck may change during treatment. ${ }^{3,4}$ Therefore, displacement errors during treatment may occur during a long treatment time such as SABR.

Two immobilization methods are currently used in our department for head and neck cancer patients. The choice of immobilization method for each patient depends on the physician's clinical considerations and preferences. One method is to use a thermoplastic mask and head support directly, and the other is to consider the variability of the patient's neck and shoulder, and then add a vacuum bag. However, the optimal fixation during treatment is unknown. Therefore, this study aimed to compare the intrafractional motion in six degrees of freedom in the two immobilization methods.

We retrospectively collected image-guidance data from recurrent head and neck cancer patients who underwent salvage SABR by CyberKnife and compared the variation trend of six axes during treatment. According to the obtained data of each axial displacement variation, the estimated planning target volume (PTV) margin was analyzed to provide a reference for clinical treatment. In addition, we further evaluated whether the safe margins of each axis were sufficient to cover the errors of each axis to reduce the uncertainty of treatment.

\section{Patients and Methods}

\section{Patients}

This study was approved by the institutional review board in our institution (No. 201801618B0). The need for informed consent from each patient was waived by the institutional review board because this study was noninvasive and utilized routine treatment data.

This study collected 9094 records from 41 patients receiving salvage SABR by CyberKnife for recurrent head and neck cancer. Forty-one patients who received SABR from November 2016 to November 2018 were enrolled. There were 23 cases of local recurrence and 18 cases of neck recurrence. The doses were prescribed to $70-92 \%$ isodose line ranging from 24 to 50 Gy in $3-6$ fractions, with a mean dose of $41.3 \mathrm{~Gy}$, and the treatment time was 20-67 minutes per fraction. Patient characteristics in these two groups were shown in Table 1.

\section{Patient Immobilization and Simulation}

All the patients were immobilized in the supine position, with a 2.4-mm-thick U-Frame thermoplastic mask and
Table I Patient Characteristics

\begin{tabular}{|c|c|c|c|}
\hline \multirow[t]{2}{*}{ Characteristics } & Group A & Group B & \multirow[t]{2}{*}{$P$ value } \\
\hline & $n=21, n(\%)$ & $n=20, n(\%)$ & \\
\hline Age (Mean, SD) & $57.9(10.7)$ & $56.6(8.7)$ & $0.67 I^{\mathrm{a}}$ \\
\hline \multicolumn{4}{|l|}{ Gender } \\
\hline Male & $18(85.7)$ & $17(85.0)$ & $1.000^{\mathrm{b}}$ \\
\hline Female & $3(14.3)$ & $3(15.0)$ & \\
\hline Initial Stage & & & $0.654^{c}$ \\
\hline IV & $13(61.9)$ & II (55.0) & \\
\hline$\leq \mathrm{III}$ & $8(38.1)$ & $9(45.0)$ & \\
\hline Collimator & & & $1.000^{\mathrm{b}}$ \\
\hline InCise MLC & $17(81.0)$ & $16(80.0)$ & \\
\hline Iris variable aperture & $4(19.0)$ & $4(20.0)$ & \\
\hline Treatment time (min) (Mean, SD) & $39.1(9.8)$ & $44.1(10.7)$ & $0.133^{\mathrm{a}}$ \\
\hline Treatment site & & & $0.890^{\mathrm{c}}$ \\
\hline Local recurrence & $12(57.1)$ & II (55.0) & \\
\hline Oral cavity & 3 & 7 & \\
\hline Nasopharynx & 1 & 0 & \\
\hline Oropharynx & 3 & 2 & \\
\hline Hypopharynx & 3 & 1 & \\
\hline Larynx & 1 & 0 & \\
\hline Other & 1 & 1 & \\
\hline Neck recurrence & $9(42.9)$ & $9(45.0)$ & \\
\hline Oral cavity & 5 & 7 & \\
\hline Nasopharynx & I & 0 & \\
\hline Oropharynx & 0 & 1 & \\
\hline Hypopharynx & 1 & 1 & \\
\hline Larynx & 2 & 0 & \\
\hline GTV $\left(\mathrm{cm}^{3}\right)$ (Mean, SD) & $94.0(79.5)$ & $160.7(144.9)$ & $0.133^{\mathrm{a}}$ \\
\hline PTV $\left(\mathrm{cm}^{3}\right)$ (Mean, SD) & 142.5 (102.2) & 209.1 (188.2) & $0.24 I^{\mathrm{a}}$ \\
\hline
\end{tabular}

Notes: ${ }^{\text {a }}$ The $P$ value calculated from independent $t$-test. ${ }^{\mathrm{b}}$ The $P$ value calculated from Fisher's exact test. 'The $P$ value calculated from Chi-square test.

Abbreviations: SD, standard deviation; MLC, multileaf collimator; min, minutes; GTV, gross tumor volume; PTV, planning target volume.

headrest (Timo Headrest, CIVCO Radiotherapy, Orange City, IA, USA). In this study, 21 patients were treated using only a thermoplastic mask and head support immobilization (Group A) (Figure 1A), and another 20 patients were immobilized for neck and shoulder with not only a thermoplastic mask and headrest but also a vacuum bag (VacQfix ${ }^{\mathrm{TM}}$ Cushion, Qfix, Avondale, PA, USA) (Group B) (Figure 1B). Vacuum bags have a durable and non-permeable Nylon outer shell that prevents punctures and tears. They are entirely airtight, filled with low-density polystyrene mini-spheres, and can be molded to the patient's back and shoulder. After the molding was completed, the patient underwent computed tomography (CT) 

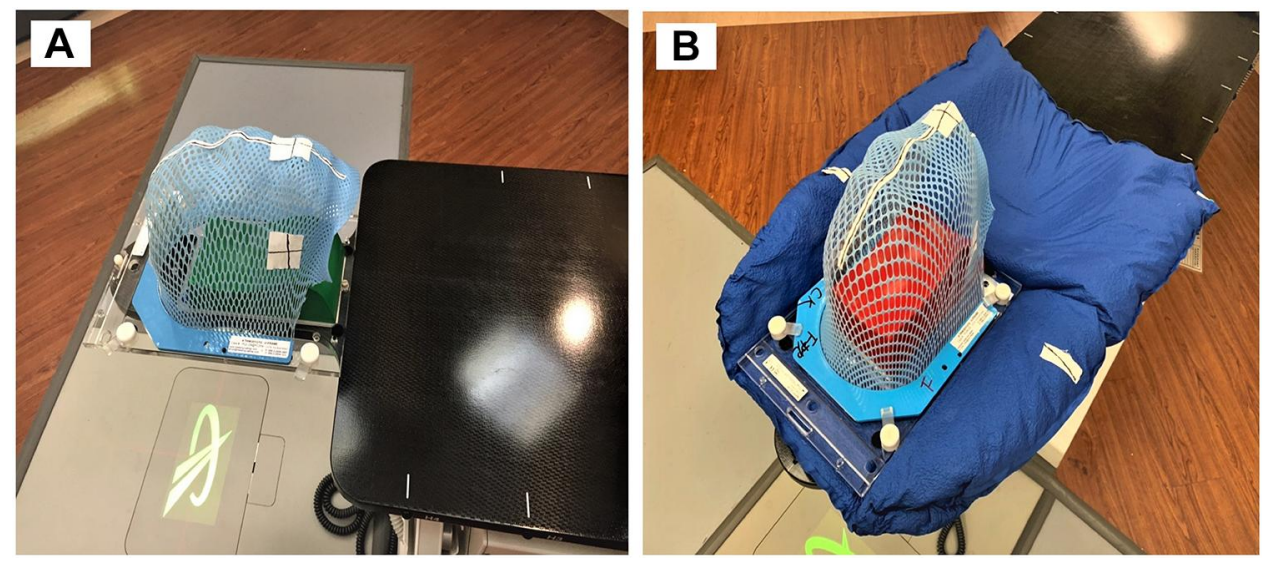

Figure I Patient Immobilization. (A) Thermoplastic Mask (Group A). (B) Thermoplastic Mask with Vacuum bag (Group B).
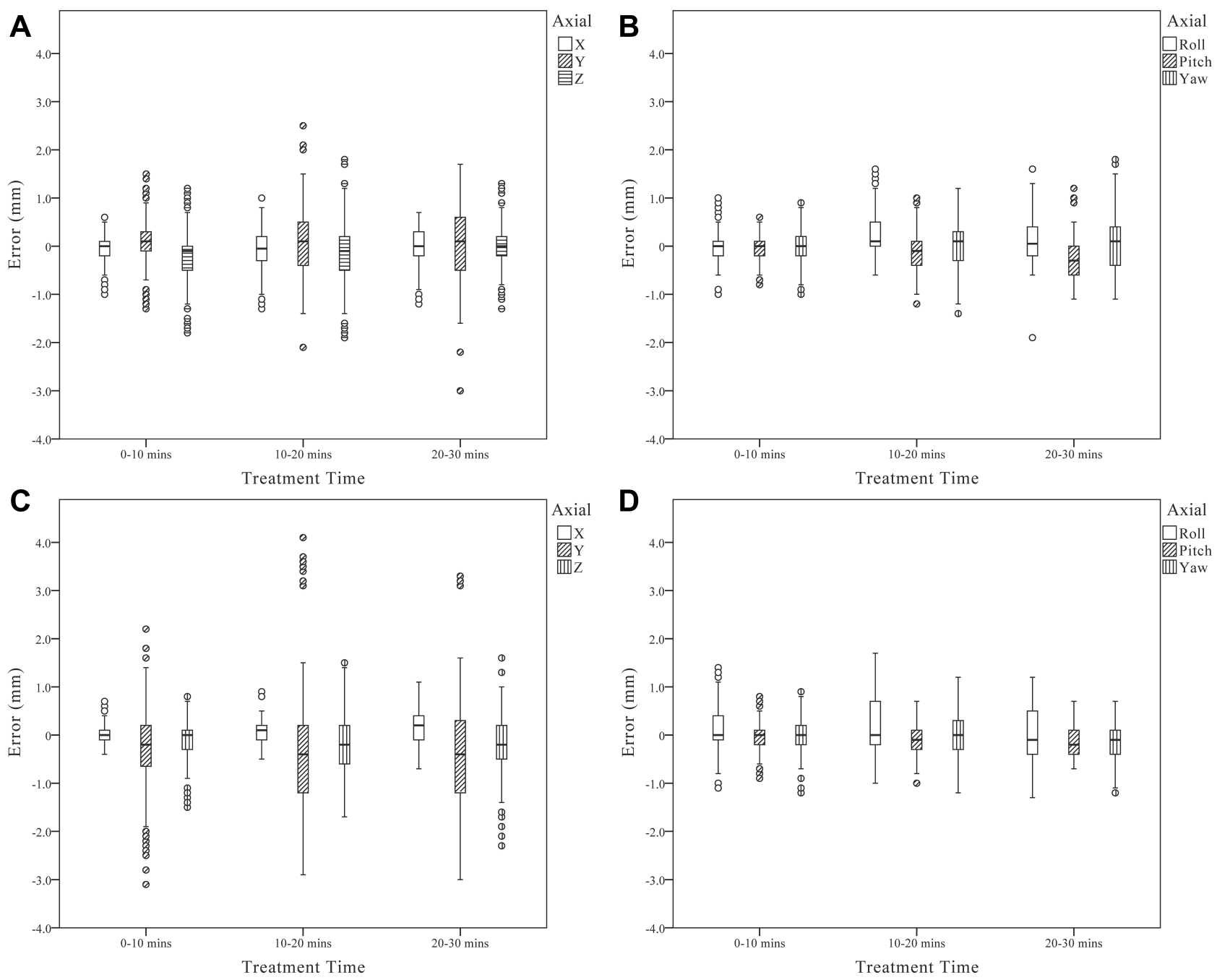

Figure 2 The box plot of the displacement error for each axis. (A) Translational errors in Group A. (B) Rotational errors in Group A. (C) Translational errors in Group B. (D) Rotational errors in Group B. 
simulation with $1.25-\mathrm{mm}$ slice thickness covering head and neck area.

\section{CyberKnife M6 System}

The CyberKnife M6 system (Accuray, Inc., Sunnyvale, CA, USA) is a novel stereotactic radiosurgery device for whole-body tumors. It utilizes a compact linear accelerator (6 MV) with a computer-controlled robotic arm to target several beams of radiation at a lesion from many different directions as a cyberknife radiosurgery system. ${ }^{5}$ It is equipped with an image guidance system (Target Locating System) that provides information about the location of the treatment target throughout the treatment. This system uses two X-ray sources mounted overhead on either side of the treatment couch. The X-ray sources provide orthogonal pairs of live X-ray images which are then compared to reference digitally reconstructed radiography (DRR) images synthesized from patient's CT to calculate the differences in the three translational and three rotational axes. Coordinates of all treatments in this study are based on the position of the patient's C-spines. The translational axes comprise the $\mathrm{X}$-axis of superior-inferior direction, the positive to the foot; the Y-axis of right-left direction, the positive to the left; the Z-axis of anteriorposterior direction, the positive to the abdomen. The rotational axes include the Roll, which is centered on the $\mathrm{X}$-axis, turning right to positive; the Pitch is centered on the $\mathrm{Y}$-axis, raising head to positive; the Yaw is centered on the Z-axis, rotating counterclockwise to positive.

\section{Xsight Spine Tracking}

Xsight spine tracking is a image guidance technique in CyberKnife. Spine tracking relies on the bony structure along the spinal column. Image registration is based on the differential contrast between bony features in the vertebral bodies. During planning, the planner defines an imaging center (Align Center) that is just anterior to the spinal cord and midline relative to the vertebral body. A region of interest containing an $81(9 \times 9)$ nodes grid is generated. This algorithm performs small-image registrations for 81 nodes at the intersections of a rectangular tracking grid.

Xsight Spine Tracking computes the offset between live X-ray images and reference DRR images by identifying and matching skeletal features. Before treatment, the patient is aligned using an adjustable couch to reduce the corrections to below the maximum robotic manipulator limits $\left(10,10,10 \mathrm{~mm}, 1^{\circ}, 1^{\circ}, 3^{\circ}\right)$. During treatment, the Xsight Spine Tracking collects real-time images every
30-45 seconds. A default interval of 30 seconds between each image acquisition is used in our hospital when the treatment starts. Then, the interval can increase gradually up to 45 seconds after verifying the stability for the first few minutes.

\section{Data Analysis and Statistics}

We analyzed the intrafractional motion in six degrees of freedom for the two immobilization methods. Of the 41 patients in this study, 22 patients did not adjust the treatment couch throughout the treatment. The data for another 19 patients were collected before the adjustment of the couch to ensure the same baseline for all records. The recording time for analysis was around 30-40 minutes, because the couch would be adjusted to compensate for the error after the actual clinical treatment lasting more than this period. Taking every 10 minutes as a time session, the average of all the images in the time session was taken to reduce the deviation of the overall result analysis caused by the extreme value measured by a single image at a specific time point, and can be completely presented as trend of displacement of the entire group.

The mean deviations of all images around each time session for each patient could be averaged to obtain the errors at each time point of six axes (X, Y, Z, Roll, Pitch, and Yaw). The absolute deviations in three translational axes at each time point for each patient were used to calculate the three-dimensional (3D) vector error as follows:

$$
3 D=\sqrt{X^{2}+Y^{2}+Z^{2}}
$$

We used independent $t$-test, chi-square test and Fisher's exact test to evaluate the difference between two groups. The result was considered statistically significant at $P<$ 0.05. All analyses used IBM SPSS Statistics 22.0 software (IBM Corp., Armonk, NY, USA).

The PTV margin (Mptv) proposed by van Herk et al. ${ }^{6}$ was estimated to ensure that $90 \%$ of the treatment plan volume was covered by $95 \%$ of the prescribed dose as follows:

$$
\mathrm{Mptv}=2.5 \Sigma+0.7 \sigma
$$

The system error $(\Sigma)$ was the standard deviation of the average of each treatment record, and the random error $(\sigma)$ was the mean square root of each treatment record average.

Finally, we also used the cumulative frequency on the translation axes for statistical analysis, and all errors were presented as absolute values. We calculated the margin of PTV to achieve a $95 \%$ cumulative frequency coverage. 
Table 2 Mean Translational and Rotational Errors Between Two Immobilization Methods

\begin{tabular}{|l|l|l|l|}
\hline \multirow{2}{*}{ Axes } & Group A & Group B & \multirow{2}{*}{ P value $^{\mathbf{a}}$} \\
\cline { 2 - 3 } & Mean (SD) & Mean (SD) & \\
\hline$X(\mathrm{~mm})$ & $-0.10(0.43)$ & $0.15(0.46)$ & $<0.00 \mathrm{I}$ \\
Y $(\mathrm{mm})$ & $0.12(0.75)$ & $-0.29(\mathrm{I} .16)$ & $<0.00 \mathrm{I}$ \\
$\mathrm{Z}(\mathrm{mm})$ & $-0.15(0.63)$ & $-0.19(0.57)$ & $0.25 \mathrm{I}$ \\
$3 \mathrm{D}$ vector & $0.92(0.58)$ & $1.12(0.88)$ & $<0.00 \mathrm{I}$ \\
Roll (degree) & $0.05(0.6 \mathrm{I})$ & $0.14(0.49)$ & 0.005 \\
Pitch (degree) & $-0.15(0.37)$ & $-0.11(0.32)$ & 0.049 \\
Yaw (degree) & $0.002(0.47)$ & $-0.05(0.44)$ & 0.027 \\
\hline
\end{tabular}

Note: ${ }^{a}$ The $P$ value calculated from independent $t$-test.

Abbreviations: SD, standard deviation; $X$, superior-inferior direction; $Y$, right-left direction; Z, anterior-posterior direction; 3D, three-dimensional.

\section{Results}

The box plots of the displacement errors for each axis in different treatment periods are presented in Figure 2. In Group A (Figure 2A and B), the median of the X, Y, and $\mathrm{Z}$ triaxial errors in different periods did not change significantly. However, the error for the Y-axis increased significantly with time. Notably, the range of variation of the Y-axis error increased as well. In the Roll, Pitch, and Yaw three-axis error median, the median error of the Pitch axis moved to a negative value with time. In Group B (Figure 2C and D), the Y-axis error data range increased with time, and the Pitch axis error median tended to be negative with time.

The mean translational and rotational errors between two immobilization methods are listed in Table 2. The translational movements in the $\mathrm{X}$-axis, $\mathrm{Y}$-axis, and $3 \mathrm{D}$ vector in Group A were significantly smaller than those in Group B. The rotational errors in the Roll and Yaw in Group A were also significantly smaller than those in Group B; conversely, those in the Pitch in Group A were larger.

The systematic errors, random errors, and estimated PTV margins proposed by van Herk et al. for the three 10- minute sessions are listed in Table 3. In Group A, the estimated margins in the $\mathrm{X}, \mathrm{Y}$, and $\mathrm{Z}$ axes were $1.68 \mathrm{~mm}, 2.80 \mathrm{~mm}$, and $1.90 \mathrm{~mm}$, respectively. In Group $\mathrm{B}$, the estimated margins were $1.35 \mathrm{~mm}, 3.78 \mathrm{~mm}$, and $2.12 \mathrm{~mm}$, respectively. As time increased, the margins gradually mildly increased in both groups.

The cumulative frequency of errors with continuous increments of $0.1 \mathrm{~mm}$ of deviation in the translational axes are plotted in Figure 3. To cover 95\% intrafractional motion, PTV margins of at least $0.96,1.55$, and $1.51 \mathrm{~mm}$ in the $\mathrm{X}, \mathrm{Y}$, and $\mathrm{Z}$ axes were needed in Group A, and 1.06, 2.86, and $1.34 \mathrm{~mm}$ were needed in Group B, respectively. The clinical use of $2 \mathrm{~mm}$ as a three-dimensional margin of PTV to cover $95 \%$ intrafractional motion was adequate in Group A but not in Group B.

\section{Discussion}

The purpose of immobilization is to ensure reproducibility of the patient's position at each treatment and improve its accuracy and stability. This study was a retrospective study to compare the intrafractional displacements between different immobilization methods. Ours is the first study to evaluate continuous intrafractional movements in the head and neck area with different immobilization methods using the Xsight spine tracking technique in CyberKnife. In this study, we used the thermoplastic mask and head support with or without a vacuum bag to assess whether adding a vacuum bag could increase stability or not during the long treatment time of SABR.

In the literature, several studies have evaluated the different head and neck immobilization methods. Lin et al. ${ }^{7}$ compared three immobilization methods in patients with nasopharyngeal carcinoma undergoing intensitymodulated radiation therapy. The immobilization methods in their study were inclusive of (a) headrest and base plate;

Table 3 The Systematic Errors, Random Errors and Estimated Margins in the 10-Min Sessions

\begin{tabular}{|c|c|c|c|c|c|c|c|c|c|c|}
\hline \multirow[t]{2}{*}{ Group } & \multirow{2}{*}{$\begin{array}{l}\text { Session } \\
(\mathrm{min})\end{array}$} & \multicolumn{3}{|c|}{$X(\mathrm{~mm})$} & \multicolumn{3}{|c|}{$\mathbf{Y}(\mathbf{m m})$} & \multicolumn{3}{|c|}{$\mathbf{Z}(\mathbf{m m})$} \\
\hline & & $\Sigma$ & $\sigma$ & $M$ & $\Sigma$ & $\sigma$ & $M$ & $\Sigma$ & $\sigma$ & $\mathbf{M}$ \\
\hline \multirow{3}{*}{ A } & $0-10$ & 0.22 & 0.15 & 0.66 & 0.42 & 0.34 & 1.30 & 0.48 & 0.31 & 1.43 \\
\hline & $11-20$ & 0.40 & 0.19 & 1.13 & 0.67 & 0.36 & 1.92 & 0.66 & 0.37 & 1.90 \\
\hline & $21-30$ & 0.38 & 0.2 & 1.09 & 0.81 & $0.4 I$ & 2.31 & 0.53 & 0.24 & 1.48 \\
\hline \multirow{3}{*}{ B } & $0-10$ & 0.31 & 0.26 & 0.96 & 0.71 & 0.71 & 2.27 & 0.32 & 0.26 & 0.97 \\
\hline & $11-20$ & 0.49 & 0.10 & 1.29 & 1.24 & 0.39 & 3.38 & 0.46 & 0.26 & 1.34 \\
\hline & $21-30$ & 0.45 & 0.31 & 1.35 & 1.21 & $0.4 I$ & 3.31 & 0.74 & 0.38 & 2.12 \\
\hline
\end{tabular}

Abbreviations: $X$, superior-inferior direction; $Y$, right-left direction; Z, anterior-posterior direction; $\Sigma$, systematic errors; $\sigma$, random errors; $M$, estimated margins. 

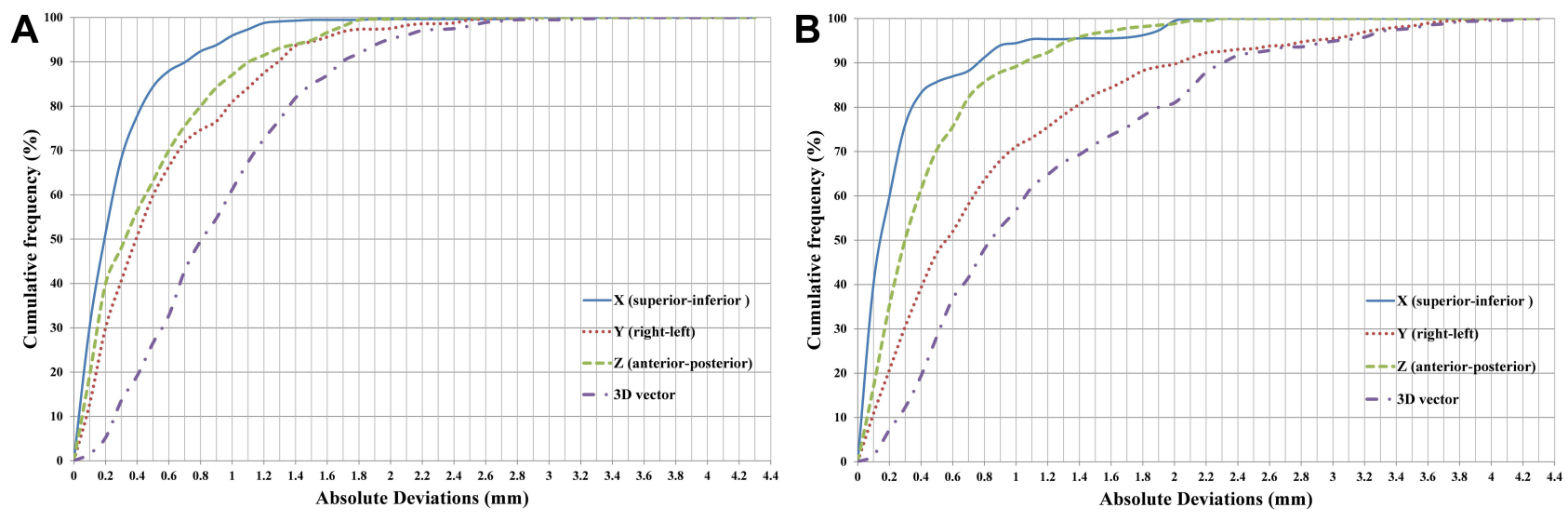

Figure 3 Cumulative frequency of translational deviations. (A) Group A. (B) Group B.

(b) alpha cradle supporting the head and shoulder; (c) vacuum bag supporting the head and shoulder. The weekly cone-beam CT (CBCT) was used to evaluate interfractional motion in these three methods. They found that the alpha cradle system was better than the others. Also, Tryggestad et al. ${ }^{8}$ compared four immobilization methods used in patients undergoing frameless intracranial radiation therapy. The immobilization methods in their study comprised (a) Type-S IMRT mask with head cushion; (b) Uni-Frame mask with head cushion and BlueBag body immobilizer; (c) Type-S head and shoulder mask and cushion; (d) Type-S head and shoulder mask and cushion, coupled with a mouthpiece. The daily pre- and posttreatment $\mathrm{CBCT}$ was used to evaluate the intrafractional deviations. They found that Type-S head and shoulder mask and cushion, coupled with a mouthpiece, had the best accuracy and stability.

In this study the treatment time was divided into three 10-minute sessions to see the changes in each axis. We found that intrafractional movements would gradually increase as time went by. The displacements of $3 \mathrm{D}$ vector, Roll and Pitch began to differ after 10 minutes of treatment, while those of X-axis, Y-axis, and Yaw began to change after 20 minutes. In the past, the PTV margin was mainly determined by the equipment, treatment site, or treatment technique, and rarely considered the displacement error caused by the treatment time. There is not much literature to discuss the influence of this aspect.

The PTV margin adopted for SABR in the head and area varied in different radiation therapy machines and institutions. Some previous studies ${ }^{9-12}$ explored small treatment volumes and their PTV was defined directly from gross tumor volume without adding any margin.
Some studies suggested that the PTV margin needed to be $2-6 \mathrm{~mm}$ in SABR. ${ }^{13-17}$ Wang et al. ${ }^{18}$ at the MD Anderson Cancer Center used a custom cushion/mask/biteblock immobilization system for SABR, and the calculated PTV margin was within $1.6 \mathrm{~mm}$. In our study, the random error $(\sigma)$ of the two groups increased with time but did not change significantly, but the systematic error $(\Sigma)$ showed significant changes with time (Table 3 ). The estimated margin could be as high as $3.78 \mathrm{~mm}$ of Y-axis in Group B.

Mesko et al. ${ }^{19}$ analyzed the influence of target sites and found that skull base sites had fewer interfractional and intrafractional errors than neck/mucosal sites. Their calculated PTV margins for skull base sites $(1.5 \mathrm{~mm})$ were smaller than those for neck/mucosal sites $(1.8-2 \mathrm{~mm})$. The treatment target sites in our study were mainly below the skull base level because we only enrolled the patients using an image guidance technique with Xsight spine tracking. For the treatment target sites around the skull base, we would adopt a 6D-skull tracking image guidance technique. We analyzed the intrafractional motions of target sites according to different image guidance alignment centers (Upper C-spine versus Lower $\mathrm{C}$-spine); however, we could not get consistent results in all the six axes to conclude which target sites have better immobility. Regardless of target sites, Iqbal et al. ${ }^{20}$ suggested an isotropic PTV margin of $3 \mathrm{~mm}$ based on the institutional experience with their immobilization method and image guidance technique. This margin could be reduced to $2 \mathrm{~mm}$ for the patients in our Group A using thermoplastic mask and headrest with Xsight spine tracking.

In our study, we found that adding a vacuum bag to the thermoplastic mask and head support did not provide 
better head and neck stability, which might be due to it potentially working on the stability of the shoulder but not the head and neck area. Regardless of the type of fixation used, the error was significantly greater in the Y-axis than the other axes. This result showed that there was still room for improvement in the Y-axis (right-left direction). We found that this shift increased with time, which might be due to involuntary head and neck movement caused by the corresponding muscle contraction and relaxation. We continue to lookfor a better immobilization method to improve setup accuracy and reduce inter- and intrafractional movement.

Our research has some limitations. First, this was a retrospective study, and there might be some selection bias in the patients enrolled. Second, the procedures of immobilization and patient setup would not be performed by the same radiation therapist at each treatment despite using the same standard operating procedures. Third, this study could only represent the result of treatment time of around 30-40 minutes of each treatment by SABR for head and neck cancer. It could not represent the results of longer treatment times.

\section{Conclusions}

The immobilization method of thermoplastic mask and head rest with a vacuum bag did not provide better immobilization than a setup without a vacuum bag in most axes. The clinical use of $2 \mathrm{~mm}$ as a margin of PTV to cover $95 \%$ intrafractional motion was adequate in Group A but not in Group B. We need to further explore better immobilization methods to improve the intrafractional stability, especially in the Y-axis (right-left direction).

\section{Abbreviations}

SABR, Stereotactic ablative radiation therapy; PTV, Planning target volume; CT, computed tomography; DRR, digitally reconstructed radiography; 3D, threedimensional; Mptv, PTV margin; CBCT, cone-beam CT.

\section{Ethics Approval and Informed Consent}

The Chang Gung Medical Foundation Institutional Review Board approved this retrospective research and waived the requirement for informed consent (No. 201801618B0). The patient data accessed complied with relevant data protection and privacy regulations.

\section{Acknowledgments}

Some contents of abstract, tables and figures were ever presented as a digital poster (\#: 2859) at 2019 ASTRO (American Society for Radiation Oncology) Annual Meeting, Chicago, USA.

\section{Funding}

This study was supported by the grants from Chang Gung Medical Foundation (CMRPG8J1071).

\section{Disclosure}

The authors declare that they have no conflicts of interests.

\section{References}

1. Jeon W, Wu HG, Song SH, Kim JI. Radial displacement of clinical target volume in node negative head and neck cancer. Radiat Oncol $J$. 2012;30(1):36-42. doi:10.3857/roj.2012.30.1.36

2. Hong TS, Tome WA, Chappell RJ, Chinnaiyan P, Mehta MP, Harari PM. The impact of daily setup variations on head-and-neck intensity-modulated radiation therapy. Int J Radiat Oncol Biol Phys. 2005;61(3):779-788. doi:10.1016/j.ijrobp.2004.07.696

3. Ezzell LC, Hansen EK, Quivey JM, Xia P. Detection of treatment setup errors between two CT scans for patients with head and neck cancer. Med Phys. 2007;34(8):3233-3242.

4. Kapanen M, Laaksomaa M, Tulijoki T, Kellokumpu-Lehtinen PL, Hyodynmaa S. Effects of remedies made in patient setup process on residual setup errors and margins in head and neck cancer radiotherapy based on 2D image guidance. Rep Pract Oncol Radiother. 2015;20 (4):292-298.

5. Bichay TJ, Mayville A. The continuous assessment of cranial motion in thermoplastic masks during cyberknife radiosurgery for trigeminal neuralgia. Cureus. 2016;8(5):e607.

6. van Herk M, Remeijer P, Rasch C, Lebesque JV. The probability of correct target dosage: dose-population histograms for deriving treatment margins in radiotherapy. Int J Radiat Oncol Biol Phys. 2000;47 (4):1121-1135.

7. Lin CG, Xu SK, Yao WY, Wu YQ, Fang JL, Wu VWC. Comparison of set up accuracy among three common immobilisation systems for intensity modulated radiotherapy of nasopharyngeal carcinoma patients. J Med Radiat Sci. 2017;64(2):106-113.

8. Tryggestad E, Christian M, Ford E, et al. Inter- and intrafraction patient positioning uncertainties for intracranial radiotherapy: a study of four frameless, thermoplastic mask-based immobilization strategies using daily cone-beam CT. Int J Radiat Oncol Biol Phys. 2011;80 (1):281-290.

9. Voynov G, Heron DE, Burton S, et al. Frameless stereotactic radiosurgery for recurrent head and neck carcinoma. Technol Cancer Res Treat. 2006;5(5):529-535.

10. Paravati AJ, Heron DE, Gardner PA, et al. Combined endoscopic endonasal surgery and fractionated stereotactic radiosurgery (fSRS) for complex cranial base tumors-early clinical outcomes. Technol Cancer Res Treat. 2010;9(5):489-498. doi:10.1177/153303461000900507

11. Dizman A, Coskun-Breuneval M, Altinisik-Inan G, Olcay GK, Cetindag MF, Guney Y. Reirradiation with robotic stereotactic body radiotherapy for recurrent nasopharyngeal carcinoma. Asian Pac J Cancer Prev. 2014;15(8):3561-3566. doi:10.7314/APJCP.2014.15.8.3561

12. Eisenhauer EA, Therasse P, Bogaerts J, et al. New response evaluation criteria in solid tumours: revised RECIST guideline (version 1.1). Eur J Cancer. 2009;45(2):228-247. doi:10.1016/j.ejca.2008.10.026 
13. Rwigema JC, Heron DE, Ferris RL, et al. The impact of tumor volume and radiotherapy dose on outcome in previously irradiated recurrent squamous cell carcinoma of the head and neck treated with stereotactic body radiation therapy. Am J Clin Oncol. 2011;34 (4):372-379. doi:10.1097/COC.0b013e3181e84dc0

14. Vargo JA, Wegner RE, Heron DE, et al. Stereotactic body radiation therapy for locally recurrent, previously irradiated nonsquamous cell cancers of the head and neck. Head Neck. 2012;34(8):1153-1161. doi:10.1002/hed.21889

15. Lartigau EF, Tresch E, Thariat J, et al. Multi institutional Phase II study of concomitant stereotactic reirradiation and cetuximab for recurrent head and neck cancer. Radiother Oncol. 2013;109 (2):281-285. doi:10.1016/j.radonc.2013.08.012

16. Kress MA, Sen N, Unger KR, et al. Safety and efficacy of hypofractionated stereotactic body reirradiation in head and neck cancer: long-term follow-up of a large series. Head Neck. 2015;37 (10):1403-1409. doi:10.1002/hed.23763
17. Wang K, Heron DE, Clump DA, et al. Target delineation in stereotactic body radiation therapy for recurrent head and neck cancer: a retrospective analysis of the impact of margins and automated PET-CT segmentation. Radiother Oncol. 2013;106(1):90-95.

18. Wang $\mathrm{H}$, Wang $\mathrm{C}$, Tung $\mathrm{S}$, et al. Improved setup and positioning accuracy using a three-point customized cushion/mask/bite-block immobilization system for stereotactic reirradiation of head and neck cancer. J Appl Clin Med Phys. 2016;17(3):180-189.

19. Mesko S, Wang H, Tung S, et al. Estimating PTV margins in head and neck stereotactic ablative radiation therapy (SABR) through target site analysis of positioning and intrafractional accuracy. Int J Radiat Oncol Biol Phys. 2020;106(1):185-193. doi:10.1016/j. ijrobp.2019.09.010

20. Iqbal MS, West $\mathrm{N}$, Richmond $\mathrm{N}$, et al. A systematic review and practical considerations of stereotactic body radiotherapy in the treatment of head and neck cancer. Br J Radiol. 2020;20200332.

\section{Publish your work in this journal}

Cancer Management and Research is an international, peer-reviewed open access journal focusing on cancer research and the optimal use of preventative and integrated treatment interventions to achieve improved outcomes, enhanced survival and quality of life for the cancer patient.
The manuscript management system is completely online and includes a very quick and fair peer-review system, which is all easy to use. Visit http://www.dovepress.com/testimonials.php to read real quotes from published authors. 